\title{
Commentary
}

\section{The evolution of anatomic pathology ${ }^{1}$}

\author{
Stanley Cohen* and Frederick Coffman \\ The Center for Biophysical Pathology, RUTGERS-New Jersey Medical School, Newark, NJ, USA
}

\begin{abstract}
Science advances both by conceptual leaps and by improved observational and analytic tools. Mechanism and function in biological systems can best be understood in the context of the complex microenvironments in which they occur, and for this purpose morphologic analysis can be critical. Technological advances in cell and tissue imaging are currently finding application in a wide variety of basic, translational, and clinical biomedical studies. "Biophotonics in Pathology" was designed as a multi-authored work to describe the various kinds of imaging strategies that have been developed as computational power keeps increasing. Some of these overlap with radiologic techniques and others do not. The field is continuously evolving, and in this commentary I will touch on additional techniques for morphology-based interrogation of cells and tissues that have recently been described. However, it is important to note that though we are expanding our armamentarium as pathologists, our radiological colleagues have been doing this for many years. Clearly, they have embraced new imaging techniques to a greater extent than have pathologists. This commentary discusses some of the factors responsible for this, and suggests that pathology and radiology are converging towards a more holistic approach to diagnostic imaging.
\end{abstract}

\section{Introduction}

Biomedical research has evolved in parallel with emerging technologies to facilitate that research. In the past, such advances have come through the application of methods based on physics and chemistry. Some examples include ultracentrifugation, spectroscopy, chromatography, electron microscopy, and fluorescence microscopy. These advances in turn lead to advances in our understanding of biology, which led to the revolution in molecular biology, which has become the new springboard for methods, techniques, and instrumentation that accelerate the pace of research even further. This is true not only in research but also in clinical medicine. The clinical pathology laboratory of today would have looked like science fiction only a decade ago. Both radiology and anatomic pathology

\footnotetext{
${ }^{1}$ Reprinted and adapted from "Biophotonics in Pathology; Pathology at the Crossroads", S. Cohen, IOS Press, pp. 325-33, 2013.

${ }^{*}$ Corresponding author: Stanley Cohen, MD, The Center for Biophysical Pathology, RUTGERS-New Jersey Medical School, Newark, NJ 07101, USA. E-mail: cohenst@umdnj.edu.
}

are based upon imaging modalities and the range of these has also expanded with our new capabilities. Radiology has taken the lead, with CAT, MRI, PET and other approaches that allow not only for finer detail and a greater range of information but also for functional analysis, even at the molecular level. This has been aided by the digital revolution, with attendant means for image enhancement and analysis, as well as rapid dissemination of this information to appropriate caregivers.

Pathology too is beginning to embrace new approaches to imaging. However, the initial object for analysis for radiologists is an image, whereas in pathology it is a tissue. In general, image-based analysis at the cellular and tissue level requires bandwidth far beyond what is necessary for radiology. Pathologists often need to work in three dimensions (Z-stacking), even with preparations of limited thickness. Cytology provides a good example of this. For these and many other reasons, anatomic pathology has not evolved as rapidly as radiology. This evolution is of critical importance because of the increasing need for the pathologist to 
provide not only diagnostic but also prognostic support as well as therapeutic guidance. Morphologic examination is no longer confined to expert pattern recognition of cells and tissues but now involves quantitative analysis that provides insights into biological behavior in general and disease mechanisms in particular. This trend is important for basic research as well. Morphologic-based investigation has become of greater importance with the realization that reactions and interactions at the molecular level do not take place in an undifferentiated "soup" within the cell, but occur in spatially distributed compartments of the cell.

Unfortunately, the technologic basis for these approaches is expanding more rapidly than our ability to exploit it for both clinical and experimental ends. While the theoretical and practical underpinnings of the new technology are complex, the underlying physical principles are straightforward. In spite of this, collaboration of engineers and physical scientists with biomedical researchers is often hampered by communication issues. Biologically oriented scientists do not feel comfortable with the concepts and approaches of the physical sciences and their mathematical underpinnings, while engineers and physicists have tended to use relatively simple, unsophisticated biological models in their work.

Because imaging techniques can take advantage of all portions of the electromagnetic spectrum (Fig. 1), it would seem that the number of approaches that are being developed is staggering. Fortunately there are only a limited number of ways for energy to interact with a biological specimen. These are absorption, reflection, scatter, diffraction, and secondary emission.

This makes the understanding of the complete array of imaging strategies somewhat easier to understand. The various publications and reviews in these areas have mainly confined themselves to a subset of these strategies that have already demonstrated their power in research and/or diagnostic settings. These include fluorescence-based techniques such as confocal microscopy not only for high resolution 3D reconstruction but also for investigation of molecular interactions, as well as several approaches to superresolution, tomographic techniques, high resolution magnetic resonance, and interferometry. Optical computed tomography has proven to be a powerful tool for in vivo analysis. Computerized image analysis is also now available for extracting maximal information from images. False color imaging is also worthy of note. This usually involves the depiction of images in color that were recorded in the visual or non-visual parts of the electromagnetic spectrum. However, false color is also useful for information visualization of data not depicting parts of the electromagnetic spectrum. Examples are elevation maps, astronomical observations, and closer to the subject at hand, magnetic resonance imaging. In particular, it is possible to display spatially correlated data from a variety of biophysical techniques as false color images or other kinds of visually interpretable mappings. This makes it easier to see

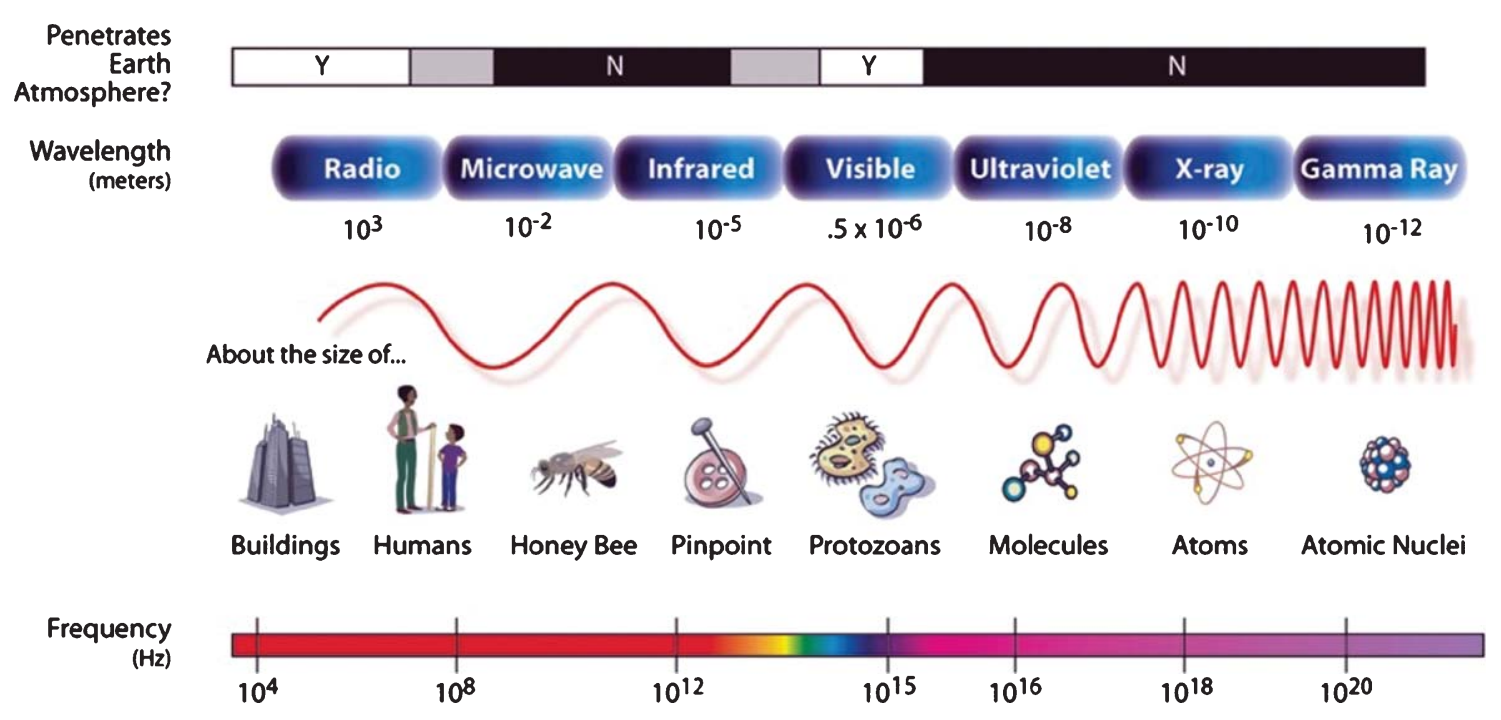

Fig. 1. The electromagnetic spectrum (NASA stock photograph). 
relationships among variables and also makes the data amenable to image analysis algorithms. Two examples of biophysical techniques that are amenable to this kind of display and analysis are impedance tomography and Raman spectroscopy.

In this regard, informatics as a discipline is no longer confined to data mining or analysis of genomic or proteomic data, but now includes all aspects of information extraction form large data sets. With the recognition that an image is a highly correlated matrix array of data points, informatics has grown to embrace image analysis. This has been facilitated by the increasing sophistication of digital imaging. Whole slide imaging is already used for frozen sections and consultations as well as archiving, and they provide a rich source of material for image quantitation and analysis. This is true not only of visual images but of any data matrix that can be displayed in the form of a visual representation.

Just as the flourishing of molecular biology has led to a paradigm shift that has rejuvenated our field, the convergence of biomedicine, bioengineering, and sophisticated new tools of mathematical analysis will move us towards a more quantitative and analytic discipline.

\section{Evolving and emerging technologies}

As indicated above, there is a substantial literature covering new cell and imaging approaches that have already proven their usefulness in basic and translational research, and have documented potential for providing diagnostic and prognostic information in a clinically relevant setting. There are many more emerging technologies, based not only on variants of these but also more novel strategies. Many of these novel approaches involve revisiting and extending existing techniques, while others represent more radical departures. I will briefly describe some of these, using examples that demonstrate the kinds of approaches being taken. This is not meant to be exhaustive, detailed, or definitive. Additionally, I will provide only one or two references, chosen mainly for accessibility to the non-specialist reader, with apologies to many others who have contributed substantially in these various fields. The purpose is to give some idea of the breadth and depth of research in this field, as well as its rapid progression. Where mention is made of specific companies and proprietary approaches, these are only meant to be representative examples of what is currently available. There is no attempt to evaluate or make recommendations of these over any I have omitted, and I have no financial interest in so doing.

\subsection{Tomographic techniques}

Photoacoustic tomography (PAT). An ultimate goal of biomedical technology is the high resolution imaging of cells and tissue in vivo. Currently, the various techniques for in vivo microscopy, such as optical computed tomography (OCT), are limited to structures at or close to the surface. For a variety of reasons, including scatter, ways of obtaining deep penetration are inversely related to resolution. While radiologic techniques utilize wavelengths capable of deep penetration, tissue is a highly scattering medium for electromagnetic waves in the optical spectral range. Thus it is possible, using these wavelengths, to obtain good images at low imaging depth in tissue or poor images with low resolution at deeper depths. Moreover, random photon paths in scatter make reconstruction of images extremely difficult by currently available algorithms.

Ultrasonic analysis, utilizing probes as external sources of acoustic stimulation, has good penetration but poor resolution as well. However, light absorption by molecules leads to thermally generated ultrasonic waves that can be received by detectors to form images [1]. Ultrasonic waves are much more scattered in tissue than electromagnetic waves, which leads to increased spatial resolution. By the use of multiple excitation wavelengths optical contrasts due to chemical composition can also be obtained. While the majority of studies utilizing PAT have been for basic biologic research, there are obvious potential applications in diagnostic pathology. For example, aside from overall improvement in resolution, if tumor cells and normal cells differ in light absorption, the ultrasonic signals would differ in intensity. A proof of concept study was performed by Heijblom et al. [2] utilizing PAT for the evaluation of breast pathology. Although only twelve cases were studied, in the ten malignant cases the PA Contrast of the abnormality was higher than the contrast on X-ray mammography. The PA Contrast was independent of breast density and was absent in the case of the two benign breast cyst cases studied. It should be noted that, in addition to the small number of cases, the study utilized patients with obvious, readily detectable lesions, which is why it is defined above 
as a poof-of-concept study. For this reason, studies on less suspicious tumors would have to be performed before an anterospective trial comparing PAT To conventional mammography in a large number of patients is performed.

There are also conceptually simpler approaches for improved visualization of structure. Two examples of these are presented below.

Cryoelectron tomography (CRYoET). A two dimensional slice of a cell or tissue represents only a random sampling of the complex information embedded in that sample. Moreover, because of the stochastic nature of both biochemical processes and their locus of activity within the cell, it is not sufficient to simply study molecular events in the absence of context about cellular compartments and inter-relationships among these. Thus, 3D reconstruction methods are being applied even to traditional modalities such as electron microscopy. For example, Plitzko et al. [3] have described the use of focused gallium ion beams as a blade to slice sample preparations. This carves away above and below a thin slice $(200-400 \mathrm{~nm})$. A series of such slices are then used to reconstruct a threedimensional profile of the specimen.

Phase-contrast tomography. Phase contrast microscopy is based on refractive index as a source of contrast and provides qualitative information about the source. Recently Yuan et al. [4] have described a strategy to utilize phase microscopy based on measurements of projections of refractive index in multiple directions in analogy to computed X-ray tomography. Angular projection phase images were obtained by beam rotation, avoiding perturbing the sample. Heterodyne laser interferometric microscopy was employed to image the phase delay by the sample and a galvanometer scanning mirror was installed to change the direction of illumination. The 3D refractive index tomogram was reconstructed from the projection phase images.

\subsection{Cellular magnetic resonance imaging}

Magnetic resonance imaging is the high resolution analog of radiographic MRI. As demonstrated by Foster et al. MRI has reached the point where in vivo detection of as few as 100 appropriately labeled cells can be detected in vivo [5]. This allows for the monitoring of cell fate over time as well as for non-invasive investigation of cellular events involved in metastasis.
Of great potential importance is the emergence of truly nanometer level resolution [6]. This methodology involves ultrasensitive magnetic resonance force microscopy (MRFM) with 3D image reconstruction to achieve resolution in the $10-90 \mathrm{~nm}$ range. In MRFM microfabricated cantilevers are used to measure tiny signals. The magnetic gradient is generated by a micrometer-scale magnetic tip, yielding a typical gradient millions of times larger than those of clinical specimens. However, the specimen needs to be in a high vacuum at cryogenic temperatures, thus limiting applicability in biological systems. Even at current levels of technology, such limitations are not absolute. For example electron microscopy has been quite successful in spite of the need for high vacuum conditions. Modifications analogous to environmental scanning electron microscopy (ESEM) that utilizes wet samples in gas may not be possible for MRFM but it is impossible to predict where technological innovation may go.

\subsection{Holographic tissue dynamics spectroscopy}

Techniques based on light scattering have found many uses as probes of cell structure. In contrast to optical coherence tomography, which uses a scanned spot of focused illumination, information obtained by angle-resolved low coherence interferometry uses a collimated beam to induce light scattering. It is also possible to apply full-field coherence gating to capture depth-gated speckle fields in a way so as to create digital holograms. The potential usefulness of the technique (holographic tissue dynamics spectroscopy) was presented by Nolte et al. [7] by studies of multicellular tumor spheroids. This technique provides not only morphologic information but also active membrane and organelle motion as well as gross cell shape changes.

\subsection{Exotic material lensing}

Lens-free microscopy is possible using computational techniques for image reconstruction and focus. There are also imaging approaches based upon metamaterials, for example the use of nanoscale phased arrays made of silicon coated with concentric rings of gold optical nanoparticles [8]. This creates a "lens" that is $60 \mathrm{~nm}$ thick. The rings impart phase delays that change the direction of the light. Thus, in this setting, phase shifts occur at the surface of the lens rather 
than through the thickness of the material as in a glass lens. The array can be tuned for specific wavelengths of light, but present technology is limited to telecom wavelengths with scalability from near infrared to terahertz. Though this is obviously in the early stages of development, it holds the promise of eliminating distortions and other artifacts that come with traditional lenses.

\section{Biophysical pathology}

As the term is currently used, biophysical pathology has two separate but related meanings. First it involves the study of biophysical parameters of cells and tissues in disease states. Second, it refers to the application of physical principles to novel ways of interrogating cells and tissues for mechanistic, diagnostic and prognostic information. The bulk of our discussion relates to the latter. However, the former involves methodologies that can lend themselves to imaging modalities and image-based computational analysis. In this section, we will briefly summarize three such approaches.

As mentioned above, it is possible to display spatially correlated data from a variety of biophysical techniques such as false color images or other kinds of visually interpretable mappings. This is especially important in that the various physicochemical processes that maintain cellular viability and function are not simply reactions among molecules that randomly interact in a homogenous fluid medium. Rather such reactions may occur on intracellular lattice surfaces, and often within discrete cellular compartments. Thus, it is likely that a pathologic process even if not associated with easily measureable molecular pathway alterations may result from or be detected by alterations in the distribution of these within the cell. This is the rationale behind the use of methodologies that can measure cellular distribution. One such example that we have investigated involves surface plasmon resonance (SPR). SPR can detect molecular rearrangements and mass redistribution within a region extending into the cell about $150 \mathrm{~nm}$ from the cell membrane. We have found [9] that cancer cells from different tissues (including breast, bone, colon, cervix and lung) have different SPR profiles. Perhaps most intriguing is the observation that, for a set of related breast cancer cell lines, there appears to be a correlation with the malignant potential of those lines.
Similar results were found when measuring impedance profiles [10]. Also, in those studies patterns of impedance response correlated with genotypic profiles. SPR is not readily amenable to mapping. Impedance tomography is feasible, but of low resolution. Raman spectroscopy is amenable to distribution mapping and commercial instruments are available from Renishaw, Horiba and others, with the potential of high spatial resolution. Raman spectroscopy can identify specific molecular species, but since scattering detects molecular vibration, especially of covalent bonds, one can utilize a cellular Raman map to look for differences in patterns of signal within cells, even in the absence of detailed chemical information.

Another potentially powerful technique that has received little attention to date is laser vibrometry, which can provide information related to both movement and structural properties such as elasticity. I am aware of no studies at the cellular or tissue level. In fact, only recently has laser vibrometry been applied to the monitoring of biological life signs at the whole body level [11] or to tissue-mimicking materials [12]. The results obtained are difficult to interpret because many motions are occurring simultaneously (involuntary micromovements, thorax, lungs, cardiac beats, etc). Moreover, the waveforms remotely captured in highly inhomogeneous environments are contaminated by multiple reflections/scattering of an interrogating transmission, as well as environmental and sensor noise. In addition, many applications significantly limit data acquisition time. All of these make conventional signal processing techniques (CSPT), such as Fourier, wavelet, and statistical analysis difficult.

When we looked, with Dr. Dmitri Donskoy at the Stevens Institute of Technology, at complex, and apparently uninterpretable thoracic vibrations, we found that the innovative Empirical Mode Decomposition (EMD) technique resulted in information that connected features derived from the captured signals to specific cardio-vascular events. The EMD is an adaptable numerical procedure for decomposing the signal. The EMD is realized through the iterative process known as "sifting". "Sifting" involves finding local maximums, minimums, interpolating the "min" and "max" envelopes with splines, determining the envelope's mean, and iterative subtracting the envelope mean from the original data until the first IMF is obtained. IMF is defined as a function satisfying two conditions: (a) in the whole data set, the number of extrema and the number of zero crossings must either 
equal or differ at most by one; and (2) at any point, the mean value of the envelope defined by the local maxima and the envelope defined by the local minima is zero. Further decomposition of the signal is carried out by subtracting the first "sifted" IMF from the original data and the "sifting" process continues until the second IMF is found. The EMD is finalized when the last IMF contains no more than two extrema. The details of this procedure can be found in [13].

Using EMD on the data captured by a scanning laser Doppler vibrometer (LDV) we were able to detect cardiac-related events, which were timed against the EKG. Vibrations and ECG were measured simultaneously. The two signals did not show an obvious correlation until we time-shifted one with respect to the other. This is shown in Fig. 2.

As Fig. 2 illustrates, $\mathrm{P}$ and $\mathrm{T}$ waves and the QRS complex match very well with respective LDV peaks. However, additional features are observed in the vibration signal measured with the LDV. These features include an extra diastolic peak between $\mathrm{T}$ and $\mathrm{P}$ wave maxima, a peak located between the QRS and the T wave, and profound ripples riding on the peak that may be associated with the $\mathrm{P}$ wave. The reason for the temporal displacement of the signals is that the electrical events trigger the mechanical effects. Without further study, no definitive explanation for the extra features is possible, although one possible hypothesis is that the diastolic peak could be attributed to mechanical vibrations in the aorta.

In any event, these whole organ results suggest that properties of isolated cells and cell monolayers might also be susceptible to analysis by laser micrometry via the use of suitable mathematical algorithms for data extraction. For example, it should be feasible to explore the microstructural mechanical characteristics of such preparations using piezoelectric cantilevers as triggering stimuli with simultaneous laser interferometry. Scanning laser microscopes for LDV measurements are commercially available for vibrometric measurements at this scale, as such instruments are used to analyze structural vibrations in nanotechnology studies. Commercial sources include Lambda Photometrics and Polytec among others.

\section{The convergence of pathology and radiology}

The fields of pathology and radiology had their beginnings in the work of Rudolf Virchow (1821-
1902) and Wilhelm Roentgen (1845-1923) respectively. With no grants, limited publication, and no institutional review, they nevertheless opened major areas of translational and basic research and forever impacted the future of diagnostic medicine. This was in spite of non-scientific obstacles rare for the current generation of biomedical scientists. For example, Virchow was once challenged to a duel by Bismarck. As the challenged party, Virchow had the choice of weapons. According to the contemporary scientific literature, he chose two sausages, one normal and one loaded with Trichinella larvae. The challenger declined the proposition. With hindsight, this may have been one of the earliest recorded examples of the importance of a negative control. Roentgen did not have such a dramatic incident. However, two weeks after his discovery of X-rays, he took the very first anatomic picture using $\mathrm{X}$-rays of his wife's hand. She became distraught and exclaimed "I have seen my death". Thus Roentgen violated Rossdale's law: "a happy wife is a happy life". History does not record the consequences.

While radiologists have taken the lead in expanding their diagnostic armamentarium beyond flat plate $\mathrm{X}$-rays by embracing advances in physics, engineering and computation, anatomic pathologists still rely mainly upon the light microscope as their primary diagnostic tool. The situation is different in the clinical laboratory setting; advances in molec-

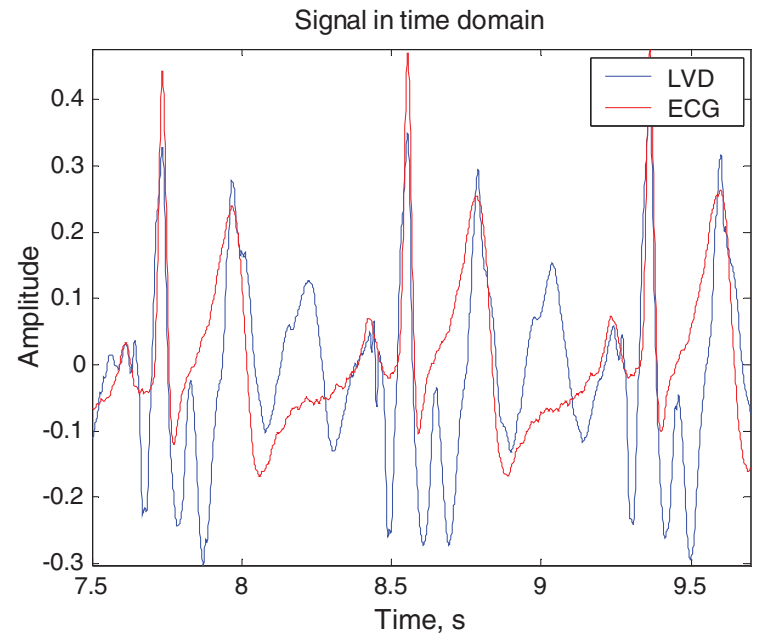

Fig. 2. LVD signals and ECG signals in the time domain. The signals were measured simultaneously, but for purpose of graphing, the LVD was shifted forward by $100 \mathrm{~ms}$. With this delay, correlations emerge between the electrical signal and the vibrational signal, as well as additional signals in the vibrational signal not present in the ECG. 
ular biology and immunology among others have greatly expanded the pathologist's ability to provide the clinician with important diagnostic information, while technological advancements have resulted in automation, high-throughput, and expanded use of data processing algorithms. Through FISH and immunohistochemistry, the expanded repertoire has reached anatomic pathology. However, it has only been recently that the initial steps towards digitalization have begun, with rapid whole slide imaging being used as an adjunct for frozen tissues and consultations. Radiologic algorithms for tomographic reconstruction, have served as an inspiration for similar approaches involving cell and tissue visualization.

There are a number of reasons why implementation of the "new pathology" has lagged behind the "new radiology". The information embedded in a tissue slice is significantly more intensive than that in a radiographic image or reconstruction, thus requiring more robust data storage and processing. This has become less of a problem as mass memory continues to become more compact and decreases in cost. Another factor is the cost of investment; for example, hospital financial administrators can more readily calculate return on investment for digital radiology than pathology; the immediate gains for pathology tend to be related to expansion of capability rather than reduction of cost. Finally, there is a chicken and egg problem. For reasons similar to those for hospital acceptance, industry has focused attention on new instrumentation for radiology more than for pathology. However, with the interest of firms such as Leica, Zeiss, Phillips, Aperio, GE, and many others in research and development of both digital pathology and new imaging modalities, research and diagnostic capability should increase as the cost of startup goes down. This does not necessarily mean that instrumentation will become less costly, but rather the availability of easy to use or complete turnkey systems will simplify the transition from conventional pathology with consequent reduction in training and implementation costs.

For these reasons, application of physical principles and computational analysis to the morphologic study of cells and tissues will continue, allowing the extraction of maximal information and functional analysis in relation to morphologic structure, in addition to breaking barriers to ultra-high resolution. Concurrently with this trend, the methodologies of radiology and pathology are beginning to overlap as are the strategies for diagnostic discovery via translational research. In addi-
The founding fathers

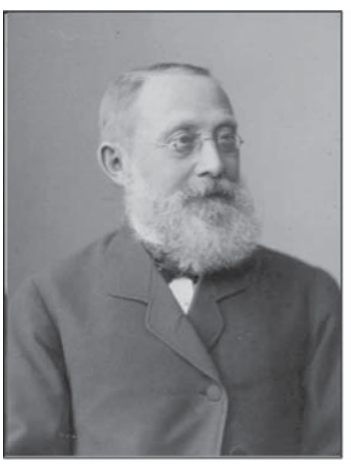

Virchow

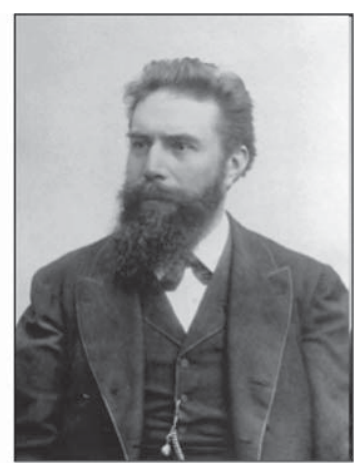

Roentgen
Fig. 3. Rudolf Virchow (1821-1902) and Wilhelm Roentgen (1845-1923).

tion, whole slide imaging technology will allow the pathologist to enjoy the same kind of digital workflow that the radiologist has become familiar with, and provides opportunities for both image enhancement and computerized image analysis. Meanwhile the new approaches to cell and tissue imaging that we are discussing have the potential to revolutionize pathology in the same way that CAT, MRI, and PET have transformed radiology. More and more, the two clinical disciplines are beginning to look similar in terms of both diagnostic strategies and methodologies in support of those strategies. This allows for a synergistic interaction for the provision of clinical information for diagnosis, prognosis, and therapeutic guidance. As such we are jointly a critical part of the transition to patient- and disease-specific personalized medicine. It may not be too much of a stretch to suggest that in the future there may be a single medical specialty, "Diagnostic Imaging" that encompasses both fields. Even today, multi-modality studies that blur the line between disciplines are appearing; for an example see [14], which describes a brain tumor molecular imaging strategy using a triple-modality MRI photoacousticRaman nanoparticle! In Fig. 3 it is no coincidence that Virchow and Roentgen are looking at one another.

\section{Summary and conclusions}

Scientific discovery and the instrumentation that facilitates such discovery are linked in that they mutually drive each other. While in the earliest stages 
of research in biology and then medicine, research was mainly based upon visual observation. Rapid progress in biology, chemistry, and physics led to more mechanistic studies involving physiologic processes, molecular interactions, and analysis at the systems level. Advances in the physical sciences are now bringing morphology back to the forefront, but for mechanistic analysis rather than just observation. These trends have also revolutionized diagnostic medicine, initially in radiology, and now beginning in pathology as well. Advances in mathematical decomposition and reconstitution, signal processing, and complex data array analysis have contributed to this trend, as has the increasing power of digital computation. Additionally, an increasing focus on integrative processes at the molecular, cellular, and organ levels has led to the application of novel biophysical techniques to the study of disease. The ability to display complex biophysical data as complex mappings allows for the application of computational image analysis to these studies as well. Many of these approaches are based upon emerging technology and all are heavily dependent on computational methods. With increasing sophistication of the tools available to both disciplines, pathology and radiology appear to be converging, certainly in their domains of interest, but possibly also in the emergence of a new specialty of "Diagnostic Imaging". From an even more futuristic point of view, advances in these areas, in conjunction with cognitive processing, nanotechnology, and rapid improvements in "Nex Gen" sequencing on the molecular front are slowly moving us towards the "tricorder" technology of the 22 nd century.

\section{References}

[1] L. Wang and S. Hu, Photoacoustic tomography: In vivo imaging from organelles to organs, Science 335 (2012), 1458-1462.

[2] M. Heijblom, D. Piras, W. Xia, J.C.G. van Hespen, J.M. Klaase, F.M. van den Engh, T.G. van Leeuwen, W. Steenbergen and S. Manohar, Visualizing breast cancer using the Twente photoacoustic mammoscope: What do we learn from twelve new patient measurements? Optics Express 20(11) (2012), 11582-11597. http://dx.doi.org/10.1364/OE.20.011582

[3] J.M. Plitzko, S. Nickell, G. Schweiker, F. Beck and W. Baumeister, Automated CryoET: State of the art and future perspectives, Micros Microanal 11 (2005), 306-307.

[4] W. Choi, C. Fang-Yen, S. Oh, N. Lue, R.R. Dasari, M.S. Feld and K. Badizadegan, Tomographic Phase Microscopy: Quantitative 3D-mapping of refractive index in live cells, Imaging \& Microscopy 10(1) (2008), 48-50.

[5] P.J. Foster, E.A. Dunn, K.E. Karl, J.A. Snir, C.M. Nycz, A.J. Harvey and R.J. Pettis, Cellular magnetic resonance imaging: In vivo imaging of melanoma cells in lymph nodes of mice. Neoplasia (2008), 207-216.

[6] M. Poggio an C.L. Degen, Force-detected nuclear magnetic resonance: Recent advances and future challenges, Nanotechnology (2010), 342001.

[7] D.D. Nolte, R. An, J. Turek and K. Jeong, Holographic tissue dynamics spectroscopy, J Biomed Optics 16(8) (2011), 087004, $1-14$.

[8] F. Aieta, P. Genevet, M.A. Kats, N. Yu, R. Blanchard, Z. Gaburro and F. Capasso, Nanoscale phased arrays: Aberration-Free Ultrathin Flat Lenses and Axicons at Telecom Wavelengths Based on Plasmonic Metasurfaces (in Press), Nano Letters.

[9] F.D. Coffman, R. Hamid, M.C. Cohen, R. Garippa and S. Cohen, Biophysical profiling of tumor cells, Analytical Cell Path 5 (2011), 225-234.

[10] F.D. Coffman, Y. Abassi, X. Wang, K. Ourfalian, M.C. Cohen and S. Cohen, Impedance profiles of breast cancer cells: Relationships with genotype and clinical behavior. In preparation.

[11] M.W. Urban, A. Alizad, W. Aquino, F. Greenleaf and M. Fatemi, A review of vibroacoustography and its applications in medicine. Curr Med Imaging Rev 7(4) (2011), 350-359.

[12] H. Yuan, B.B. Guzina, S. Chen, R.R. Kinnick and M. Fatemi, Estimation of the comple shear modulus in tissue-mimicking materials from optical vibrometry measurements. Inverse Problems in Science and Engineering 20(2) (2012), 173-187.

[13] N.E. Huang, Z. Shen, S.R. Long, M.C. Wu, E.H. Shih, Q. Zheng, C.C. Tung and H.H. Liu, The Empirical Mode Decomposition Method and the Hilbert Spectrum for Non-Stationary Time Series Analysis, Proc, R Soc Lond A454 (1998), 903-995.

[14] M.F. Kircher, A. de la Zerda, J.V. Jokerst, C.L. Zavaleta, P.J. Kempen, E. Mittra, K. Pitter, R.Huang, C. Campos, F. Habte, R. Sinclair, C.W. Brennan, I.K. Mellinghoff, E.C. Holland and S.S. Gambhir, A brain tumor molecular imaging strategy using a new triple-modality MRI-photoacoustic-Raman nanoparticle. Nature Medicine 18(5) (2012), 829-834. 


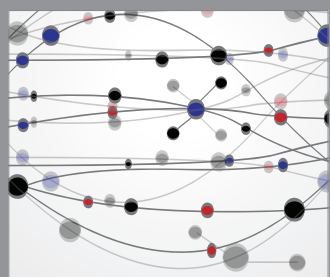

The Scientific World Journal
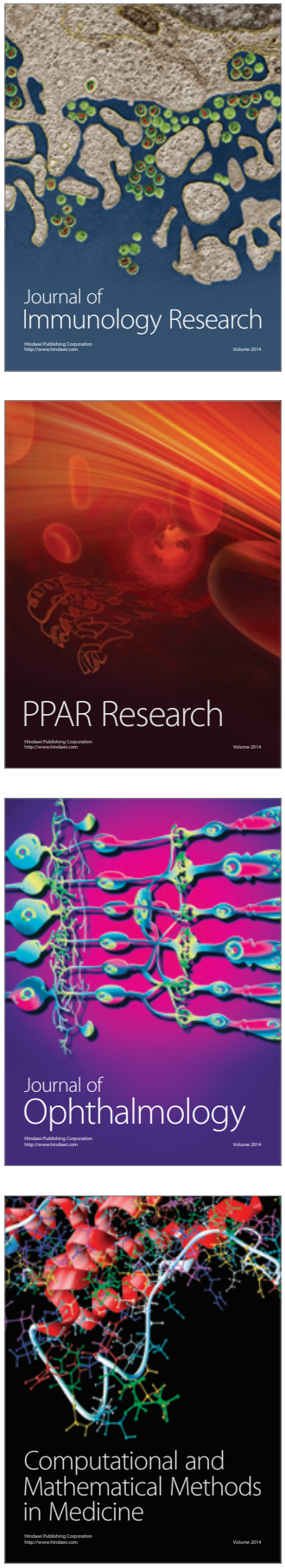

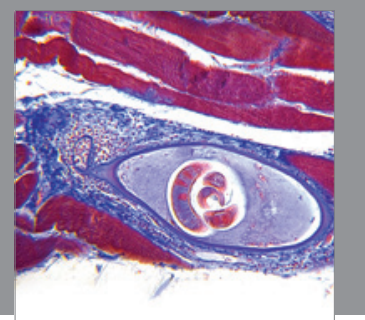

Gastroenterology

Research and Practice
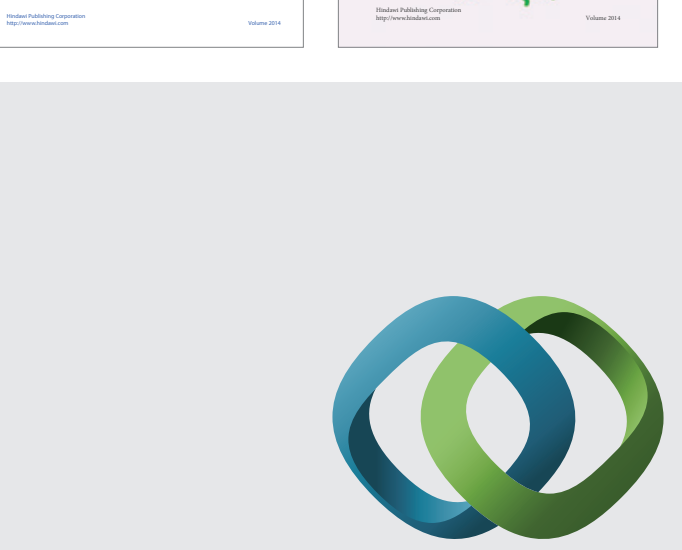

\section{Hindawi}

Submit your manuscripts at

http://www.hindawi.com
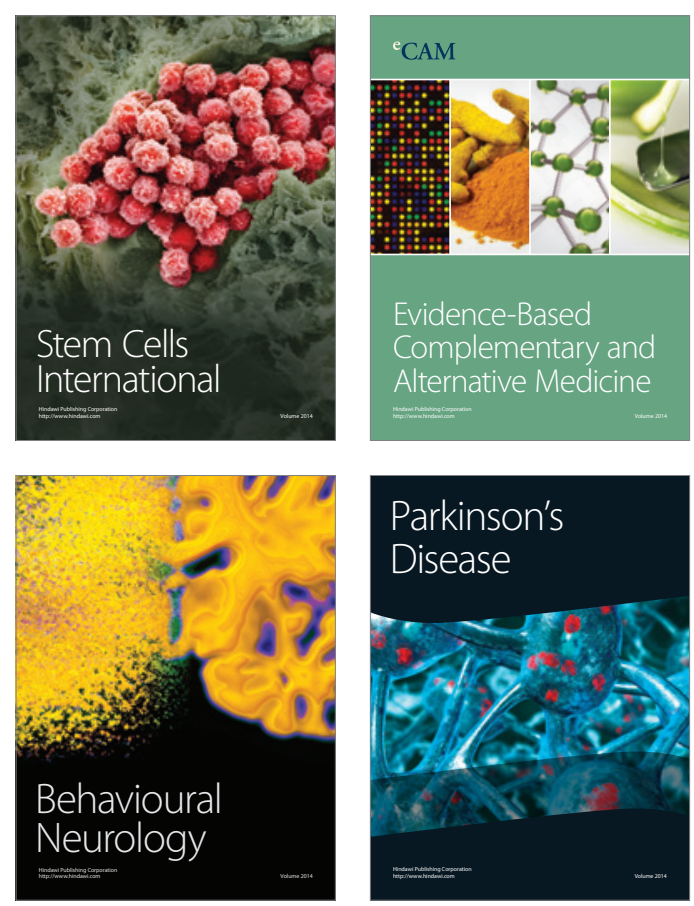

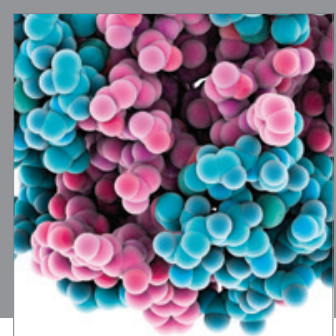

Journal of
Diabetes Research

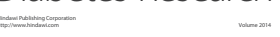

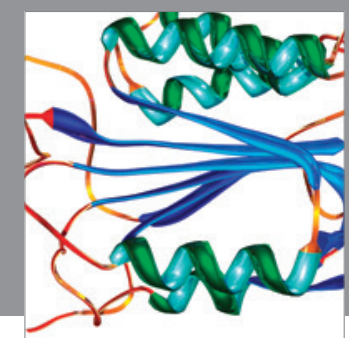

Disease Markers
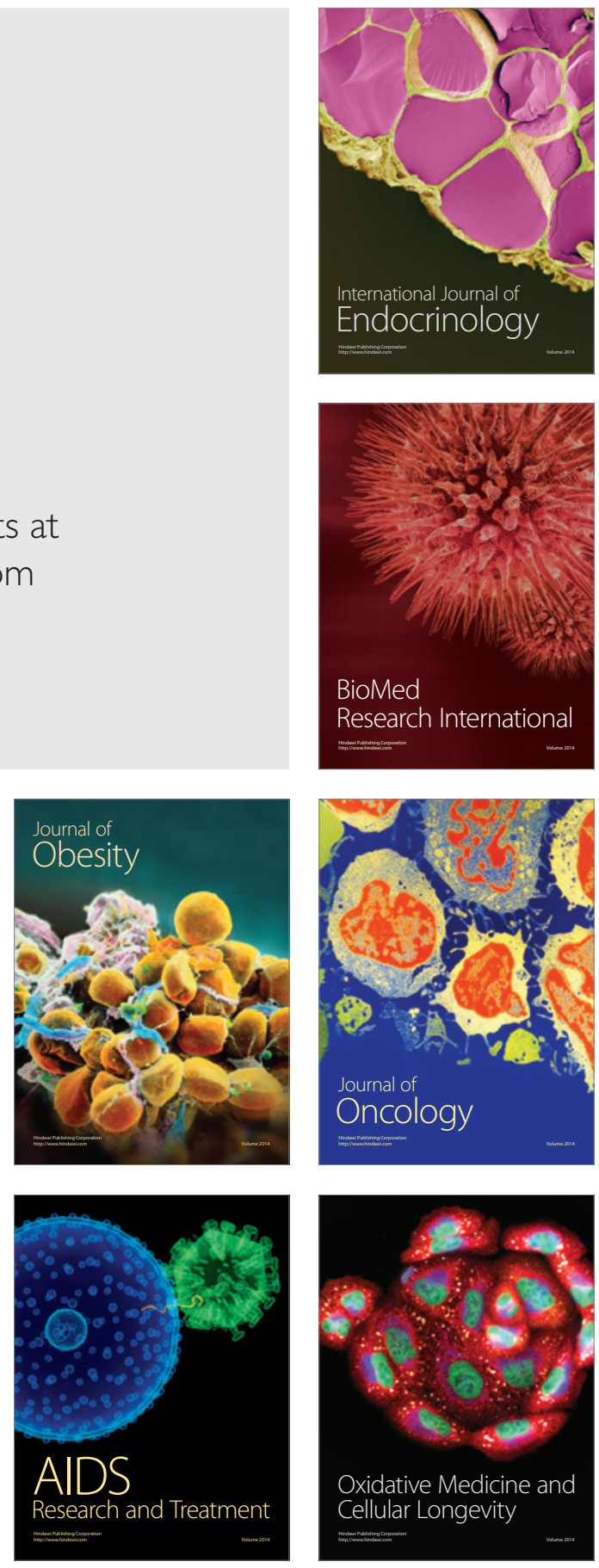